\title{
Calidad de Vida en el Trabajo y Condiciones de Trabajo, en Auxiliares de Esterilización
}

\author{
QUALITY OF LIFE AT WORK AND WORKING CONDITIONS IN AUXILIARY STERILIZATION
}

\author{
Diemen Delgado', María de los Ángeles Aguilera², Fabián Delgado³, Irma Cano ${ }^{4}$ Óscar Ramírez ${ }^{4}$ \\ 1. Departamento de Salud Ocupacional, Clínica Río Blanco, Los Andes, Chile. \\ 2. Departamento de Salud Pública, Universidad de Guadalajara, Guadalajara, México. \\ 3. Clínica del Trabajador, Manta, Ecuador. \\ 4. Escuela de Postgrado, Universidad Científica del Sur. Lima. Perú.
}

\begin{abstract}
RESUMEN
Evaluar el conjunto de factores relativos a las condiciones del trabajo que pueden tener repercusiones sobre la salud y la calidad de vida en el trabajo de las auxiliares de esterilización. Se realizó un estudio transversal y analítico, se aplicó el instrumento CVT-GOHISALO (Instrumento para evaluar calidad de vida en el trabajo), el cual mide calidad de vida en el trabajo y el método LEST (Laboratoire de Economie et Sociologie du Travail) de evaluación global ergonómica de las condiciones de trabajo; para el análisis de los datos se utilizó el programa estadístico SPSS 18 y el software online para ergonomía del trabajo de la Universidad Politécnica de Valencia. En la dimensión soporte institucional para el trabajo, las auxiliares de esterilización no visualizan oportunidades de progreso en el puesto o función que desempeñan; en la dimensión seguridad en el trabajo, sienten injusticias en las oportunidades de ascenso al interior de la institución; en la dimensión integración al puesto de trabajo, están motivadas para superar retos y muy dispuestas a ser líderes; en la dimensión satisfacción por el trabajo, suelen ser comprometidas con la misión de la institución; en la dimensión del bienestar logrado a través del trabajo, perciben riesgo en la salud física y mental; en la dimensión de desarrollo personal, son optimistas y de trato amable; en la dimensión administración de tiempo libre, cumplen plenamente con su horario y las tareas encomendadas sin menoscabo de sus compromisos personales y sociales. La condición de trabajo en cuanto a postura, manipulación de equipo quirúrgico y herramientas presenta riesgo inminente de fatiga. Las auxiliares de esterilización muestran una serie de percepciones subjetivas que reflejan una realidad de su medio laboral, que deberían inducir a la jefatura en la toma de decisiones en pro de mejorar los procesos de trabajo y de esta manera disminuir los factores de riesgos que se evidencian en este estudio, los cuales podrían producir enfermedades físicas y mentales si no se corrigen a tiempo.
\end{abstract}

(Delgado D, Aguilera M, Delgado F, Cano I, Ramírez 0, 2013. Calidad de Vida en el Trabajo y Condiciones de Trabajo, en Auxiliares de Esterilización. Cienc Trab. Sep-Dic; 15 [48]: 148-151).

Palabras claves: CALIDAD DE VIDA EN EL TRABAJO, CONDICIÓN DE TRABAJO, TRABAJADORES DE LA SALUD.

\section{ABSTRACT}

Assessing all the factors relating to working conditions that may impact on health and quality of life at sterilization assistant work. A cross-sectional analytical study was carried out, the CVT-GOHISALO instrument, which measures quality of life at work and ergonomic method LEST overall assessment of working conditions was applied, for data analysis statistical program was used SPSS 18 software for online and work ergonomics of the Polytechnic University of Valencia. In the institutional support for work dimension, sterilization assistants displayed no opportunities for advancement on the job or role, in the security dimension at work, feel injustice in promotion opportunities within the institution, in the dimension integration into the workplace, are motivated to overcome challenges and very willing to be leaders, in dimension job satisfaction tend to be committed to the mission of the institution, in the dimension of being achieved through the work, perceived risk physical and mental health in the size of personal development, are optimistic and friendly service, in the dimension of time management, fully comply with your schedule and the tasks without compromising their personal and social commitments. The working conditions in terms of posture, and manipulating surgical tools and equipment present imminent risk of fatigue. Sterilization assistants show a series of subjective perceptions that reflect the reality of their work environment, which should lead to the leadership in decision making towards improving work processes and thus reduce the risk factors that are evident in this study, which could result in physical and mental illness if not corrected in time.

Key words: QUALITY OF LIFE AT WORK, WORKING CONDITION, HEALTH WORKERS.
Correspondencia / Correspondence:

Diemen Delgado

Av. Santa Maria 777, Los Andes, Chile

e-mail: diemen.delgado@clinicarioblanco.cl

Fono: (34) 2590500

Recibido: 26 de Noviembre 2013 / Aceptado: 13 Diciembre 2013

\section{INTRODUCCIÓN}

Las centrales de esterilización como proveedoras de servicios constituyen elementos de gran importancia en el ámbito sanitario al estar directamente relacionados con el ámbito quirúrgico. ${ }^{1}$ La principal misión de una central de esterilización de un servicio sanitario es proporcionar a todos las unidades el material o equipamientos en condiciones idóneas de esterilidad. Este proceso comienza cuando el artículo es desmontado, seguido por la limpieza, inspección, emba- 
lado, etiquetado y la esterilización, para garantizar el rendimiento y la seguridad del mismo. ${ }^{2}$

No obstante pueden existir riesgos asociados a la propia actividad e independientes del tipo de esterilización que se lleve a cabo, como pueden ser la manipulación de cargas o los movimientos repetitivos ${ }^{3}$, los diferentes procesos para conseguir esterilidad conllevan una serie de peligros inherentes a la propia naturaleza del proceso que van desde quemaduras asociadas a las altas temperaturas hasta el carácter cancerígeno y mutágeno de algunos de los agentes esterilizantes químicos. ${ }^{4-7}$

Por lo tanto, teniendo en cuenta la importancia y la complejidad de esta práctica y la escasez de publicaciones sobre el tema, se realizó este estudio, cuyo objetivo fue evaluar el conjunto de factores relativos a las condiciones del trabajo que pueden tener repercusiones sobre la salud y la calidad de vida en el trabajo de las auxiliares de esterilización, más aun si se enfatiza que la calidad de vida en el trabajo ${ }^{8-11}$ es una filosofia que dignifica al trabajador y su desarrollo personal.

\section{MÉTODO}

Se realizó un estudio transversal y analítico para identificar la calidad de vida en el trabajo y las condiciones de trabajo de las auxiliares de esterilización de Clínica Río Blanco de la ciudad de Los Andes, Chile, durante el primer semestre del 2013. Se aplicó el instrumento CVT-GOHISALO, el cual consta de 74 items y mide la calidad de vida en el trabajo en siete dimensiones: soporte institucional para el trabajo, seguridad en el trabajo, integración al puesto de trabajo, satisfacción por el trabajo, bienestar a través del trabajo, desarrollo personal y administración tiempo libre. ${ }^{12}$

Para evaluar la condición de trabajo, se utilizó el método LEST de evaluación global ergonómica de las condiciones de trabajo perteneciente al Laboratorio de Economía y Sociología del Trabajo de Francia ${ }^{13}$; este método evalúa: la carga física, entorno físico, carga mental, aspectos psicosociales y tiempo de trabajo. Para el análisis de los datos, se utilizó el programa estadístico SPSS 18 y el software online para ergonomía del trabajo de la Universidad Politécnica de Valencia: Ergonautas.com

Se informó a cada participante del estudio el objetivo específico del mismo. No se realizó ninguna intervención o modificación intencionada en los individuos. ${ }^{14} \mathrm{El}$ autor, se comprometió a manejar la información bajo las normas establecidas por el Código Internacional de Ética Médica. ${ }^{14}$

\section{RESULTADOS}

El servicio de esterilización de Clínica Río Blanco cuenta con un área de recepción de material usado, área de lavado de material, área de preparación de material, área de autoclaves, área de equipo de óxido de etileno, desinfección de alto nivel, bodega de ropa y área de entrega de material. Estas tareas son realizadas por dos auxiliares de esterilización, quienes reciben el material médico quirúrgico proveniente de todos los servicios clínicos, lavan, secan, clasifican, preparan, esterilizan y anotan en un libro todo el material que ingresa y egresa del servicio; estas tareas son supervisadas por una enfermera coordinadora. A continuación se presentan los resultados del estudio.

Respecto a la calidad de vida en el trabajo, calificado de acuerdo al instrumento CTV-GOHISALO, se obtuvieron las siguientes interpreta- ciones: en la dimensión soporte institucional para el trabajo, las auxiliares de esterilización se consideran personas con actividades o tareas claras y acordes con su motivación; no obstante, no visualizan oportunidades de progreso en el puesto o función que desempeñan. En la dimensión seguridad en el trabajo, consideran sus actividades laborales como un medio para desarrollarse tanto a nivel personal como social; no obstante, sienten injusticias en las oportunidades de ascenso al interior de la institución.

En la dimensión integración al puesto de trabajo, las auxiliares de esterilización suelen ser personas cooperadoras, con facilidad para trabajar en equipo con alta complejidad, están motivadas para superar retos y muy dispuestas a ser líderes. En la dimensión satisfacción por el trabajo, suelen ser comprometidas con la misión de la institución. Son personas que muestran los aspectos positivos del trabajo, se autovaloran con orgullo por pertenecer a la institución. Los reconocimientos obtenidos son escasos o no existen.

En la dimensión del bienestar logrado a través del trabajo, las auxiliares de esterilización perciben riesgo en la salud física o emocional, se sienten insatisfechas con la remuneración adquirida por su trabajo y, por consecuencia, responsabilizan el entorno laboral por su precariedad. En la dimensión de desarrollo personal, tienen buen estado de ánimo, son optimistas y de trato amable. Trasmiten a los demás su entusiasmo; no obstante, pueden considerar que el trabajo les ha disminuido su capacidad fisca y emocional. En la dimensión administración de tiempo libre, planifican y distribuyen sus actividades laborales con las recreativas y de descanso, cumplen plenamente con su horario y las tareas encomendadas sin menoscabo de sus compromisos personales y sociales.

En cuanto a las condiciones de trabajo utilizando el método LEST, se obtuvieron los siguientes resultados. Ver Tabla I. En cuanto a la carga física, podemos dividirla en dos estados: la primera, la carga estática, que depende de la postura más frecuente adoptada por las trabajadoras, que fue la de pie, con inclinación de tronco (30 minutos/hora), seguida a la de pie con los brazos en extensión frontal (15 minutos/ hora); y, la segunda, la carga dinámica, que depende del esfuerzo realizado en el puesto de trabajo; esta resultó ser breve pero repetida (44 veces/hora), consiste en la manipulación de equipo quirúrgico y herramientas relacionadas con el proceso mismo de esterilización. El peso de la carga que provoca el esfuerzo no supera los $2 \mathrm{Kg}$ (Kilogramos), el recorrido para alimentar la máquina con materiales para esterilizar es mayor a 3 metros, con una frecuencia de transporte menor a 10/hora, el peso de trasporte es menor de $12 \mathrm{Kg}$. La condición de trabajo en cuanto a postura presenta riesgo de fatiga, mientas que en la manipulación de equipo quirúrgico y herramientas la fatiga ya está instalada; en cuanto al esfuerzo realizado en el puesto de trabajo y de aprovisionamiento existen débiles molestias

Entorno físico, el ambiente térmico es constante durante el turno de 8 horas diarias, con una temperatura promedio de 25 grados Celsius y humedad de $40 \%$. Las trabajadoras están sometidas siempre a un mismo nivel constante de ruido, que no sobrepasa los $65 \mathrm{db}$ (Decibeles) -están ausentes los ruidos impulsivos-. El nivel de atención requerido por la tarea es muy elevado, debido a una mayor necesidad de exactitud del proceso. Se trabaja con luz artificial permanentemente, no existen deslumbramientos, el nivel de iluminación en el puesto de trabajo es de 1592 lux, el de la sala de esterilización de 342 lux, el nivel de contraste en el puesto de trabajo es medio, el nivel de percepción requerido en la tarea es extremadamente fino. El nivel de vibraciones durante las 8 horas de trabajo es menor a 2 horas y son poco molestos. La condición de trabajo en cuanto a ambiente térmico, ruido y vibraciones es satisfactoria. No 
obstante, el ambiente luminoso podría mejorase y aportar más comodidad al trabajador.

En cuanto a la carga mental, el nivel de atención del trabajo es repetitivo, en cadena, sin pausas, requiere de supervisión. Si se producen retrasos en la tarea estos deben recuperarse durante el trabajo, el tiempo en alcanzar el ritmo normal de trabajo es menor o igual a hora, el modo de remuneración es un salario fijo mensual. El nivel de atención requerido por la tarea es muy elevado, debe ser mantenido por más de 40 minutos/hora, debido a una mayor necesidad de exactitud del proceso e intercambio de palabras durante el proceso de limpieza, inspección, embalado, etiquetado y esterilización. El tiempo es mayor a 5 minutos/hora en que la trabajadora puede apartar la vista del proceso dado al nivel de atención requerido, la falta de atención podrían desencadenar accidentes serios; no obstante, es raro que ocurran. La complejidad está basada en la duración media de la limpieza, inspección, embalado, etiquetado que es mayor a 16 segundos, la duración media de cada ciclo es de 5 minutos y menor de 7 minutos. La condición de trabajo en cuanto a la presión de tiempos que conlleva la exactitud de manipulación de equipo quirúrgico y herramientas podría mejorase y aportar más comodidad al trabajador; sin embargo, el nivel de atención ya está generando riesgo de fatiga.

Respecto a los aspectos psicosociales, en cuanto a iniciativa podemos observar que el trabajador no puede modificar el orden del proceso que realiza; este depende del ritmo de la cadena organizada bajo estrictas normas de calidad: controla las piezas, realiza retoques eventuales. La trabajadora influye totalmente y positivamente sobre la calidad del producto, es decir, total imposibilidad de cometer errores; en caso de producirse un incidente debe intervenir el propio trabajador. Las máquinas y equipos ocupados durante el proceso de esterilización son reguladas por el propio trabajador. Respecto a la comunicación con los demás trabajadores, el número de personas visibles por la trabajadora en un radio de 6 metros es de 2 personas (compañera y supervisora), la trabajadora no puede ausentarse de su puesto de trabajo, no existe ninguna restricción sobre el derecho a hablar, existe la posibilidad técnica de hablar en el puesto de trabajo, intercambios de palabras; en cuanto al intercambio de palabras con otros puestos de trabajo, es poco frecuente. Existe expresión obrera organizada con varios delegados muy activos. En cuanto a la relación con el mando, existen consignas al comienzo de la jornada y, a petición del trabajador, el mando no se encuentra cerca o presente frecuentemente. El puesto de la trabajadora es independiente. Respecto al status social, la duración del aprendizaje del trabajador para el puesto de trabajo es de 1 a 3 meses, la formación requerida mínima es técnico-profesional en esterilización. La condición de trabajo en cuanto a iniciativa, comunicación y relación de mando presentan débiles molestias, algunas mejoras podrían aportar más comodidad al trabajador.

Tabla 1.

Condiciones de trabajo utilizando el método LEST.

\begin{tabular}{|c|c|c|c|c|c|c|c|c|c|}
\hline $\begin{array}{l}\text { Carga } \\
\text { Física }\end{array}$ & ${ }^{*} P$ & $\begin{array}{l}\text { Entorno } \\
\text { Fisco }\end{array}$ & ${ }^{*} P$ & $\begin{array}{l}\text { Carga } \\
\text { Mental }\end{array}$ & ${ }^{*} \mathrm{P}$ & $\begin{array}{l}\text { Aspectos } \\
\text { Psicosociales }\end{array}$ & *P & $\begin{array}{l}\text { Tiempos } \\
\text { de Trabajo }\end{array}$ & ${ }^{*} P$ \\
\hline $\begin{array}{l}\text { Carga } \\
\text { Estática }\end{array}$ & 7 & $\begin{array}{l}\text { Ambiente } \\
\text { Térmico }\end{array}$ & 1 & $\begin{array}{l}\text { Presión } \\
\text { de Tiempo }\end{array}$ & 5 & Iniciativa & 4,17 & $\begin{array}{l}\text { Cantidad } \\
\text { de Tiempo }\end{array}$ & 5 \\
\hline $\begin{array}{l}\text { Carga } \\
\text { Dinámica }\end{array}$ & 8 & Ruido & 0 & Atención & 7 & $\begin{array}{l}\text { Comuni- } \\
\text { caciones }\end{array}$ & 3 & $\begin{array}{l}\text { Organización } \\
\text { Del Tiempo }\end{array}$ & 9 \\
\hline & & Iluminación & 3 & Complejidad & 0 & $\begin{array}{l}\text { Relación con } \\
\text { El Mando }\end{array}$ & 5 & & \\
\hline & & Vibraciones & 0 & & & $\begin{array}{l}\text { Status } \\
\text { Social }\end{array}$ & 0 & & \\
\hline
\end{tabular}

El horario de trabajo es de 8 horas diarias, es decir 45 horas semanales, con lo cual no existe posibilidad de horas extraordinarias, los retrasos a la hora de ingreso son imposibles; en relación de la hora de finalizar la jornada solo se puede cesar el trabajo a la hora prevista; en cuanto a las pausas durante el trabajo es imposible fijar duración y tiempo. Con relación al tiempo de descanso hay posibilidad durante el horario de colación de acuerdo al reglamento interno institucional. La condición de trabajo en cuanto a la cantidad de tiempo para cumplir con el proceso mismo de esterilización podría mejorarse y aportar más comodidad al trabajador; no obstante, la ausencia de pausas y el cumplimiento del proceso sistemático respecto a la organización del tiempo han desencadenado fatiga.

\section{DISCUSIÓN}

Este estudio constituye el primer intento para identificar la calidad de vida en el trabajo y las condiciones de trabajo en las auxiliares de esterilización. Una de las limitaciones en el desarrollo de nuestro estudio fue la escasa información relacionada a la investigación de la calidad de vida en el trabajo y las condiciones de trabajo de las auxiliares de esterilización. En cuanto a la calidad de vida en el trabajo, las auxiliares de esterilización muestran una serie de percepciones subjetivas que reflejan una realidad de su medio laboral. ${ }^{9}$

Respecto a la condición de trabajo en cuanto a carga estática esta se debió a la inclinación de tronco al realizar la limpieza de material quirúrgico; caso contrario ocurrió en otro estudio al evaluar los puestos de trabajo en el área de tapas de una empresa metalúrgica ${ }^{15}$, encontrando que la carga estática estuvo influenciada por la postura inadecuada de los brazos por encima de los hombros al momento de empaquetar bolsas.

En nuestro estudio el esfuerzo realizado en el puesto de trabajo presenta riesgo de fatiga específicamente por el movimiento repetido al momento de realizar la limpieza del material quirúrgico; en otro estudio, sobre un modelo simple para la evaluación integral del riesgo a lesiones músculo-esqueléticas ${ }^{3}$, se encontró que en condiciones de reposo, cualquier posición corporal exige un compromiso cardiovascular y energético, en la medida que transcurren las horas de trabajo.

En nuestro estudio el nivel de ruido no sobrepasa los $65 \mathrm{~dB}$ y fue calificado como situación satisfactoria. En otro estudio, al evaluar los puestos de trabajo de la industria pesquera del Ecuador ${ }^{16}$, encontraron que el factor que contribuye a la fatiga de los trabajadores es causado por el ruido fluctuante de alrededor de los $82 \mathrm{~dB}$, que a pesar de no sobrepasar el límite recomendado de $85 \mathrm{~dB}$ para 8 horas de trabajo, ocasiona molestias.

En nuestro estudio el nivel de iluminación y contraste provocaron débiles molestias; en otro estudio, al evaluar los factores que inciden en la astenopía de los trabajadores de inspección visual en la industria electrónica ${ }^{17}$, encontraron que la iluminación existente en los puestos de trabajo provoca fatiga visual, probablemente porque no cuenta con un buen contraste entre objeto y superficie en las tareas de inspección visual.

En nuestro estudio, la auxiliar de esterilización presenta probabilidades de alteración en la esfera de la salud mental; en otro estudio, al realizar una revisión de la literatura sobre el exceso de trabajo y agravios mentales en trabajadores de la salud ${ }^{18}$, se concluyó que el trabajo realizado de manera excesiva favorece significativamente los agravios a la salud psíquica y mental.

Otro autor realizó un análisis sobre el riesgo en el trabajo y salud 
ocupacional ${ }^{19}$, concluyendo que las principales causas de los accidentes se debian a distracciones, descuidos, despistes o falta de atención (45\%), trabajar muy rápido (19,4\%), y al cansancio o fatiga $(17,8 \%)$. Llama la atención que en nuestro estudio es raro que ocurran accidentes, a pesar que sus procesos son sistemáticos, de precisión y que la carga mental mostró riesgo de fatiga.

En nuestro estudio la iniciativa presentó molestias débiles, debido probablemente a la adquisición de un patrón relacionados a estándares de calidad en el proceso mismo de esterilización; en otro estudio, al evaluar los puestos de trabajo en el área de tapas de una empresa metalúrgica ${ }^{15}$, se observó que la iniciativa en el ayudante operador presentó una nocividad importante y en el caso del mecánico operador, solo molestias débiles; esta situación se debió principalmente a las diferencias de cargos y actividades desempeñadas por los operarios.

Otro autor realizó un análisis etnográfico sobre la complejidad y opacidad de las tareas en auxiliares de esterilización ${ }^{20}$, encontrando grados de invisibilidad general de todo el servicio. En nuestro estudio esta percepción es similar, encontrando que el intercambio de palabras con otros puestos de trabajo es poco frecuente y que presenta grados de invisibilidad.

En un estudio de caso, referente a los modos de interacción en los procesos de trabajo respecto a la relación de mando ${ }^{21}$, se observó que la coordinadora tiene un modo dual de presencia y actuación, como agente individual y coordinadora; es parte de la comunidad de prácticas, dado que ayuda, colabora y sustituye a otras trabajadoras en sus tareas en todo el proceso de esterilización. Caso contrario ocurre en nuestro estudio, donde la coordinadora no se encuentra cerca de los procesos y no participa en ellos.

En nuestro estudio, al evaluar el tiempo de trabajo este presentó riesgo de fatiga; a pesar que el proceso está determinado por un tiempo razonable, existe la percepción de las trabajadoras de que debiera incrementarse el número de auxiliares. Es importante señalar que en este servicio no existe posibilidad de realizar horas extras y que probablemente la fatiga esté relacionada con el trabajo breve pero repetitivo. Igual resultado se presentó en otro estudio al evaluar el tiempo de trabajo en cuanto a la fatiga; sin embargo, en esta investigación la fatiga se relacionó a la mayor demanda de horas extras y en muchas ocasiones no había posibilidades de rechazarlas, ya que estaban supeditadas hasta el término de la producción planificada. ${ }^{16}$

Finalmente, a la luz de los resultados, se sugiere diseñar un programa de intervención para modificar las condiciones de trabajo, eliminando los factores que podrían presentar daños físicos y mentales a mediano plazo, y con ello mejorar la calidad de vida en el trabajo, contrastando este estudio con las dimensiones evaluadas mediante el instrumento CVT-GOHISALO.

\section{Agradecimientos}

A mi madre Betty Aracely García Pinoargote por comprender el espacio del ser y el tiempo y, en la distancia, sentirla cerca de mi pensamiento y con ello poder profundizar en la dimensión de la investigación cualitativa. A mi padre Eustorgio Bernaldo Delgado Palma, que con este legado de inspiración, iniciará la huella perpetua en la historia del conocimiento, de las ciencias de la salud en el trabajo.

\section{Lista de abreviaciones / List of Abbreviations}

CVT-GOHISALO: Instrumento para evaluar calidad de vida en el trabajo. LEST: Laboratoire de Economie et Sociologie du Travail. KG: Kilogramos. DB: Decibeles. LUX: Nivel de iluminación. P: Puntaje.

\section{REFERENCIAS}

1. Jiménez ML. Formación de pre y post grado de enfermería en esterilización. Rev Enferm CyL. 2013;5(1):50-57.

2. Sánchez P, Ortiz J, Somoza E, Bernal JL, Paniagua JL Grupo de Expertos. Unidad central de esterilización: estándares y recomendaciones. Madrid: Ministerio de Sanidad Política Social e Igualdad; 2011.

3. Manero A, Soto L, Rodríguez T. Un modelo simple para la evaluación integral del riesgo a lesiones músculo-esqueléticas (MODSI). Mapfre Medicina. 2005;16(2):12-20.

4. Rivero $E$, Piñero $S$, González $S$, Briceño $A$, De Souza $L$, Bello $M$, et al. Frecuencia de uso de óxido de etileno en áreas de esterilización de centros asistenciales de Valencia y sus efectos a la salud. Rev Fac Cienc Salud. Univ Carabobo. 2006; 10(3):15-18.

5. Caamaño A, Castro F. Exposición laboral al todavia insustituible al $100 \%$ óxido etileno en unidad de esterilización. Gestión Práctica de Riesgos Laborales. 2011;86:30-37.

6. Rivero $E_{1}$ Piñero $S$, González $S$, De Souza $L$, Rodríguez $M$, Barrio $E_{1}$ et al. Aductos de hemoglobina y micronúcleos en trabajadores hospitalarios expuestos a óxido etileno. Venezuela. Salud Trab. 2009;17(1):7-22.

7. León $N$, Arteaga A. Exposición al óxido de etileno del personal auxiliar en odontología. Acta Odontol Venez. 2008;46(4):1-11.

8. Novich M. La transforación de la organización del trabajo.En: De la Garza E. Tratado Latinoamericano de Sociología de Trabajo. México: El Colegio de México-FLACSO-UAM-Fondo de Cultura Económica; 2000.

9. Delgado D, Inzulza M, Delgado F. Calidad de vida en el trabajo: Profesionales de la salud de Clínica Río Blanco y Centro de Especialidades Médicas. Med Segur Trab. 2012;58(228):216-223.

10. Delgado D, Aguilera MA, Delgado F, Rug A. The experience of miners relocated to alternative positions due to silicosis in the Andean of CODELCO, Chile, 2010. Saf Health Work. 2012;3:140-145.

11. Delgado D, Salazar J, Aguilera MA, Delgado F, Parra L, Ramírez O, González
R. Efectos en el tiempo de la reubicación laboral y la calidad de vida en trabajadores mineros con silicosis de la División Andina-Codelco, Chile. Med Segur Trab. 2011;57 (225):339-347.

12. González R, Hidalgo G, Salazar JG, Preciado ML. Instrumento para medir la calidad de vida en el trabajo (CVT-GOHISALO): Manual para su aplicación y su interpretación, México: Ed. de la Noche; 2009.

13. Guélaud F, Beauchesne MN, Gautrat J, Roustan G. Pour une analyse des conditions de travail ouvrier dans l'entreprise. Paris: A. Colin; 1975.

14. Mazzanti MA. Declaración de Helsinki, principios y valores bioéticos en juego en la investigación médica con seres humanos. Revista Colombiana de Bioética. 2011;6(1):125-144.

15. Gasca MA, Rengifo $M$, Rodríguez E. Evaluación ergonómica de los puestos de trabajo en el área de tapas de una empresa metalúrgica. Ingeniería Industrial Actualidad y Nuevas Tendencias. 2008;1(1):31-42.

16. Torres T, Rodríguez M. Evaluación ergonómica de puestos de trabajo de la industria pesquera del Ecuador. Rev Tecnol ESPOL. 2007;20(1):139-142.

17. Solano H. Ergoftalmología: Análisis de los factores que inciden en la Astenopía de los trabajadores de inspección visual en la industria electrónica de la Ciudad de Juárez. Cienc Trab. 2006;8(21):135-140.

18. Cruz ML, Chaves M, Barcellos RCM, Almeida L, De Oliveira I, Pedrao L. Exceso de trabajo y agravios mentales a los trabajadores de la salud. Rev Cubana Enfermer. 2010;26(1):52-64.

19. Gil-Monte P. Riesgo psicosocial en el trabajo y salud ocupacional. Rev Perú Med Exp Salud Pública. 2012;29(2):237-241.

20. Lozares C, Verd J, Barranco O, Massó M, Moreno S. La complejidad y opacidad de las tareas auxiliares en los procesos de trabajo: un análisis etnográfico comparado en dos servicios hospitalarios. Cuadernos de Relaciones Laborales. 2013;31(1):221-242.

21. Lozares C. La unidad de análisis, sus agentes y contextos y los modos de interacción en los procesos de trabajo: un estudio de caso. Papers. 2007;83:97-122. 